\title{
Clinical characteristics, CT signs, and pathological findings of Pyrrolizidine alkaloids-induced sinusoidal obstructive syndrome: a retrospective study
}

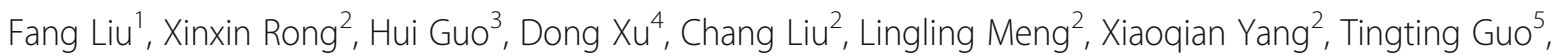 \\ Xuefeng $\mathrm{Kan}^{5}$ and Yuhu Song ${ }^{2^{*}}$ (D)
}

\begin{abstract}
Background: One major etiology of hepatic sinusoidal obstruction syndrome (HSOS) in China is the intake of pyrrolizidine alkaloids (PAs). Since PAs-induced HSOS is a rare disease that has not been clearly characterized until now, the aim of this study was to investigate clinical characteristics, CT features, and pathological findings of PAinduced HSOS.

Methods: This retrospective cohort study included 116 patients with PAs-induced HSOS and 68 patients with Budd-Chiari syndrome from Jan 2006 to Sep 2016. We collected medical records of the patients, and reviewed image features of $C T$, and analyzed pathological findings.

Results: Common clinical manifestations of PAs-induced HSOS were abdominal distention (98.26\%), ascites (100\%), jaundice (52.94\%), abdominal pain (36.36\%). Abnormal liver function was observed in most of PAs-induced HSOS. On CT scan, common findings included: ascites, hepatomegaly, the thickening of gallbladder wall, pleural effusion, patchy liver enhancement, and heterogeneous hypoattenuation. Most of the patients had a low ascitic total protein $(<25 \mathrm{~g} / \mathrm{L})$ and a high SAAG $(\geq 11.0 \mathrm{~g} / \mathrm{L})$. In acute stage, pathologic features were massive sinusoidal dilatation, sinusoidal congestion, the extravasation of erythrocytes, hepatocellular necrosis, the accumulation of macrophages, the deposition of hemosiderin. In subacute stage, complete loss of pericentral hepatocytes, sinusoidal dilatation, the deposition of pigment granules were observed.
\end{abstract}

Conclusions: The PAs-induced HSOS patients displayed distinct clinical characteristics, imaging features, and pathological findings, which provided some evidences for the diagnosis of PAs-induced HSOS.

Trial registration: ChiCTR-DRD-17010709.

Keywords: Hepatic sinusoidal obstruction syndrome, Pyrrolizidine alkaloid, Clinical manifestations, Ascites, Histology

\section{Background}

Hepatic sinusoidal obstruction syndrome (HSOS), also known as veno-occlusive disease (VOD), is characterized by damage to sinusoidal endothelium $[1,2]$. A central pathogenic event is toxic destruction of hepatic sinusoidal/central venous endothelial cells, and then the sloughed sinusoidal lining cells embolize downstream and obstruct sinusoidal

\footnotetext{
* Correspondence: yuhusong@yahoo.com; yuhusong@163.com ${ }^{2}$ Division of Gastroenterology, Union Hospital, Tongji Medical College, Huazhong University of Science and Technology, Wuhan 430022, China Full list of author information is available at the end of the article
}

flow $[3,4]$. It results in the necrosis of hepatocytes and portal hypertension. In North America and Western Europe, HSOS occurs most commonly in the patients who have received cytoreductive therapy prior to hematopoietic stem cell transplantation (HSCT), or oxaliplatin-containing chemotherapy for colorectal carcinoma [3-6]. While, the intake of pyrrolizidine alkaloids (PAs)-containing herbals or dietary supplement is a major etiology of HSOS in China [7-10]. To date, more than 6000 plant species containing PAs have been identified [11]. One of the most widely used herbals containing PAs is gynura segetum (ie Tsuanqi) in 
China [12-14].In view of the difference in the etiology of HSOS, clinical profiles and imaging findings of HSOS associated with HSCT or oxaliplatin might be hardly extrapolated to PAs-induced HSOS. In addition, PAs-induced HSOS is a rare disease and clinical manifestations of PAsinduced HSOS resemble other liver diseases. To get a better understanding of PAs-induced HSOS, a large sample of the patients (116 cases) with PAs-induced HSOS and the controls (68 patients with Budd-Chiari syndrome) were enrolled and relevant data were collected. Then, clinical profiles, CT features, and pathological finding of PAs-induced HSOS were investigated in our study.

\section{Methods}

\section{Study population}

In this study, we collected 116 patients with PAs-induced HSOS at Union Hospital and Tongji Hospital affiliated to Tongji Medical College, Huazhong University of Science and Technology (HUST) from Jan 2006 to Sep 2016. For PAsinduced HSOS, diagnostic criteria were (i) the patients met the modified Seattle criteria for HSOS (at least 2 of the following: hyperbilirubinemia ( $>34.2 \mu \mathrm{mol} / \mathrm{L}$ ), hepatomegaly or right upper quadrant pain, ascites and $>2 \%$ weight gain due to fluid accumulation) (ii) meeting the criteria for druginduced liver injury [Roussel Uclaf Causality Assessment Method (RUCAM) score > 5] [15, 16]; (iii) a history of ingestion of PAs [13, 14, 17-20]. A history of ingestion of PAs is essential in defining PAs-induced HSOS. All the enrolled patients ingested PAs-containing gynura segetum (ie Tsuanqi) in our study. Other possible etiologies of liver injury, such as viral, alcohol, drug, nonalcoholic fatty liver disease, BuddChiari syndrome (BCS), congestive heart diseases, autoimmune liver diseases, were evaluated and excluded carefully (Fig. 1).

PAs-induced HSOS patients should be differentiated from HSCT-related HSOS, or oxaliplatin-induced HSOS. Fortunately, the incidence of HSCT-related HSOS, or oxaliplatininduced HSOS is very low in China, and medical history provides a definite evidence for differential diagnosis. Importantly, PAs-induced HSOS should be discriminated from Budd-Chiari syndrome (BCS). Thus, 68 patients with BCS were enrolled in our study (Fig. 1). Budd-Chiari syndrome is defined as hepatic venous outflow obstruction at any level from the small hepatic veins (HVs) to the junction of the inferior vena cava (IVC) and the right atrium, regardless of the cause of obstruction [1, 2, 21]. Digital subtraction angiography was performed in the enrolled BCS patients. Approval for this retrospective study was obtained from our college ethics committee, and the requirement for informed consent was waived.

\section{Data collection}

The pertinent data of the patients were extracted from the medical records and tabulated in a database, which included the information about demographic data; presenting symptoms and signs; medical history; laboratory tests (blood test, ascitic examination); pathological changes, therapeutic process.

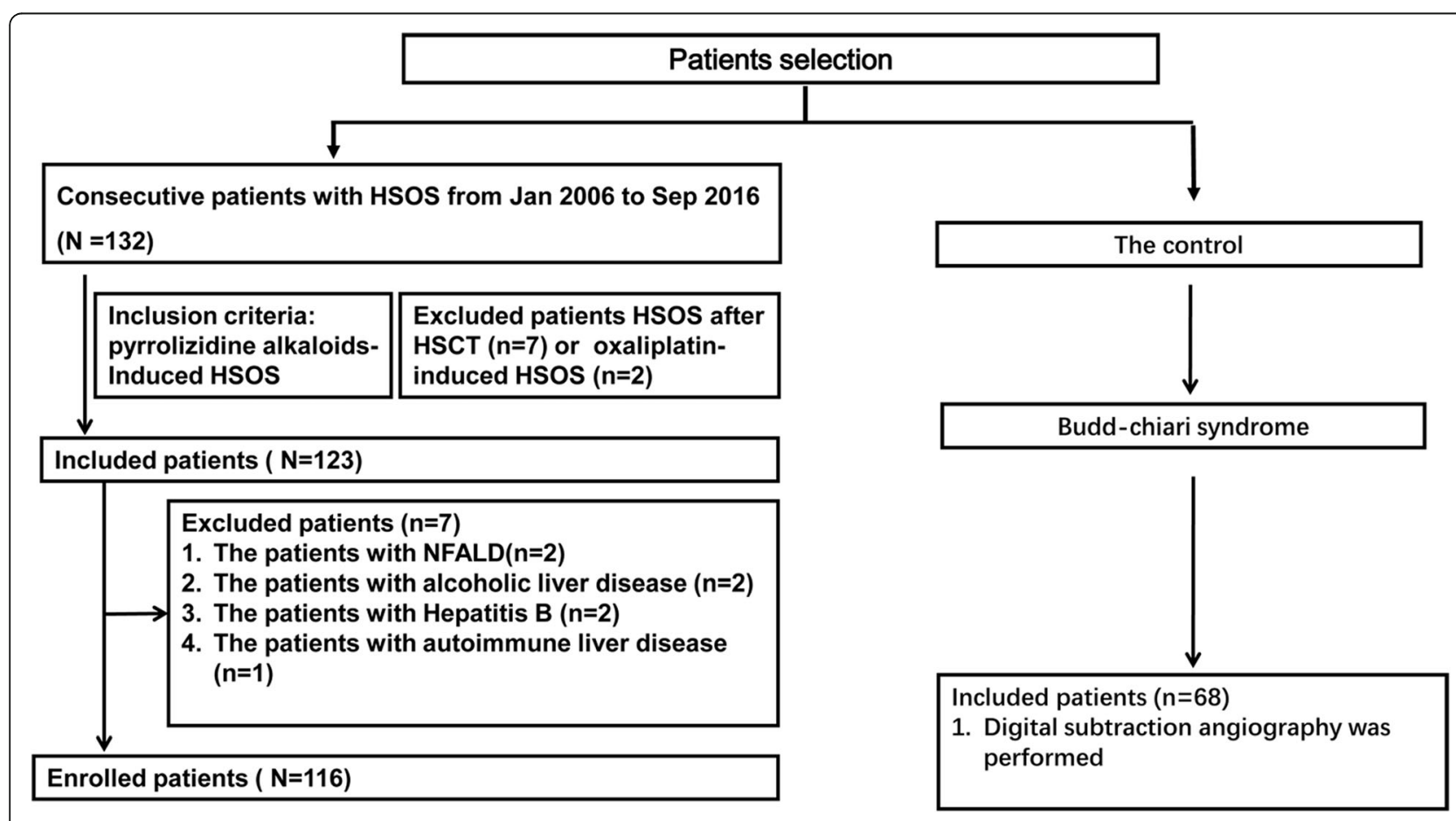

Fig. 1 Flowchart of the patients' enrollment 


\section{Imaging technique and imaging analysis}

All CT examinations were performed with one of the following three scanners: 64-detector spiral row scanner (Somatom Definition AS, Siemens, Germany), 32detector row dual-source CT scanner (Somatom Definition, Siemens, Germany), 320-detector row dynamic volume CT (Aquilion ONE 640, Toshiba, Japan). Contrast-enhanced CT was performed after the injection of contrast medium (iohexol, GE Healthcare Co. Ltd., China; lopromide, Bayer Healthcare Co. Ltd., China; Loversol, Hengrui Medicine Co. Ltd., China). Images were obtained in arterial, portal venous, and equilibrium phases, respectively, with $25-30 \mathrm{~s}, 55-70 \mathrm{~s}$, and $90-110 \mathrm{~s}$ after the injection of contrast medium. All CT images were reviewed in consensus by two experienced observers blinded to clinical data.

\section{Animal models of PAs-induced HSOS}

All animals were maintained under specific pathogenfree conditions at Laboratory Animal Center, Huazhong University of Science and Technology, China. Animals were housed individually in open ventilated cages with distilled water ad libitum until study started. Room temperature was monitored and maintained at $20-26^{\circ} \mathrm{C}$ with the light cycle set at $12 \mathrm{~h}$. Monocrotaline (MCT)treated rats, senecionine-treated mice were used as experimental models of PAs-induced HSOS [22-24]. Male Sprague Dawley rats (weight: 200-220 g) were obtained from Laboratory animal center, Huazhong University of Science and Technology (Wuhan, China). For MCTtreated rats, the rats $(n=32)$ were fasted $12 \mathrm{~h}$, and then the rats were gavaged with monocrotaline $(90 \mathrm{mg} / \mathrm{kg}$, Sigma Aldrich, St. Louis, MO, $n=16)$ or PBS $(n=16)$ $[22,25,26]$. C57BL/6 male mice were purchased from Beijing Vital River Laboratory Animal Technology Co. Ltd. (Beijing, China). In mouse model of senecionine- induced HSOS, male C57BL/6 mice (8-week-old, $n=32$ ) were fasted $12 \mathrm{~h}$, and then the mice were gavaged with senecionine $(100 \mathrm{mg} / \mathrm{kg}$, Cayman chem., Ann Arbor, Michigan, $n=16)$ or $\operatorname{PBS}(n=16)[18,23,24,27]$. All animals were euthanized by barbiturate overdose. All the animals have received human care in compliance with the institutional animal care guidelines.

\section{Statistical analysis}

Continuous variables were presented as means and standard deviation and categorical variables as numbers and percentage. Independent-samples t-test was used for the analysis of differences between the two groups. The interobserver agreement for assessment of imaging findings was determined using the $\mathrm{k}$-statistic. The level of agreement was defined as follows: poor, $k<0.20$; fair, -values of $0.2<\kappa \leq 0.40$; moderate, $0.4<\kappa \leq 0.60$; good, $0.6<\kappa \leq 0.80$; and very good, $0.8<\kappa \leq 1.0$. A $P$ value less than .05 was considered to indicate statistical significance. Statistical analyses were performed using SPSS version 17.0 (SPSS Inc., Chicago, Illinois, USA).

\section{Results \\ Clinical presentation}

In this study, 116 patients with a diagnosis of PAsinduced HSOS were enrolled in our study. The mean ages of the PAs-induced HSOS patients were $56.92 \pm$ 12.39 years. In PAs-induced HSOS patients, there were 78 males and 38 females, with a male-to-female ratio of 2.05:1. It indicated that the patients with older age and male gender were frequently affected. Then, clinical manifestations of these patients were evaluated. $98.26 \%$ $(113 / 115)$ of the cases had abdominal distension, a small proportion $(36.36 \%, 40 / 110)$ of the PAs-induced HSOS patients had right upper quadrant pain. Meanwhile, our study revealed edema in $39.45 \%$ (43/109) of the cases, jaundice in $52.94 \%(54 / 102)$ and weight gain in $15.53 \%$ $(16 / 103)$ of the patients. While, $69.12 \%(47 / 68)$ of the BCS patients had abdominal distension, right upper quadrant pain in $19.12 \%(13 / 68)$ of the cases, edema in $38.24 \%(26 / 68)$ of the cases.

\section{Laboratory tests (blood routine examination and clinical biochemistry)}

Table 1 summarized laboratory tests including blood routine examination and clinical biochemistry at the time of baseline evaluation. Firstly, blood routine

Table 1 Baseline characteristics of the patients and laboratory tests

\begin{tabular}{llll}
\hline Variables & PAs-HSOS & BCS & $P$ \\
\hline Erythrocytes, $10^{12} / \mathrm{L}$ & $5.00 \pm 4.33$ & $4.33 \pm 0.84$ & 0.053 \\
Hemoglobin, g/L & $135.99 \pm 26.81$ & $128.11 \pm 24.00$ & 0.061 \\
Leukocyte, $10^{9} / \mathrm{L}$ & $6.90 \pm 2.81$ & $4.87 \pm 2.59$ & $<0.001$ \\
Platelet, $10^{9} / \mathrm{L}$ & $114.06 \pm 63.48$ & $119.37 \pm 83.98$ & 0.651 \\
ALT, U/L & $134.5 \pm 154.89$ & $40.60 \pm 64.75$ & 0.002 \\
AST, U/L & $146.31 \pm 156.30$ & $49.72 \pm 54.81$ & 0.001 \\
ALP, U/L & $170 \pm 106.89$ & $122.25 \pm 69.08$ & 0.003 \\
Y-GT, U/L & $160.52 \pm 114.56$ & $106.60 \pm 85.94$ & 0.002 \\
T-BIL, $\mu$ mol/L & $65.07 \pm 78.83$ & $35.58 \pm 28.47$ & 0.001 \\
Albumin, g/L & $30.71 \pm 5.50$ & $35.51 \pm 6.29$ & $<0.001$ \\
PT, S & $17.43 \pm 2.64$ & $15.63 \pm 2.01$ & $<0.001$ \\
Urea, mmol/L & $7.19 \pm 3.63$ & $5.26 \pm 2.76$ & 0.001 \\
Cr, $\mu$ mol/L & $90.65 \pm 45.61$ & $64.16 \pm 18.67$ & $<0.001$ \\
\hline Note Baselne &
\end{tabular}

Note: Baseline characteristics of PAs-induced HSOS refer to initial examination during first visit to our hospitals. PAs-HSOS PAs-induced HSOS; BCS Budd-Chiari syndrome; normal ranges: erythrocytes: $3.0-5.5 \times 10^{12} / \mathrm{L}$; hemoglobin: $110-160$ g/L; leukocyte: $4-10 \times 10^{9} / \mathrm{L}$; platelet: $100-300 \times 10^{9} / \mathrm{L}$; alanine aminotransferase: 5-35 U/L; aspartate aminotransferase (AST):8-40 U/L; alkaline phosphatase (ALP) 40-150 U/L; total bilirubin (T-BIL): 5.1-19 $\mu \mathrm{mol} / \mathrm{L} ; \mathrm{\gamma}$-glutamyl transpeptidase $(\gamma-\mathrm{GT}) 7-32 \mathrm{U} / \mathrm{L}$; albumin: $35-55 \mathrm{~g} / \mathrm{L}$; prothrombin time (PT): $11-$ $16 \mathrm{~S}$; urea: $3.2-7.1 \mathrm{mmol} / \mathrm{L}$; creatinine $(\mathrm{Cr}): 44-106 \mu \mathrm{mol} / \mathrm{L}$. 
examination of the patients was investigated. Generally, the values of erythrocyte and leukocyte were in normal limit in the PAs-induced HSOS patients (Table 1); the value of platelet was lower than the lower limit of normal in $46.81 \%$ of the patients. Then, the parameters of clinical chemistry including a panel of liver functional tests and renal function were evaluated. The median levels of liver enzymes (AST, ALT, ALP and GGT) and total bilirubin exceeded upper limit of normal range in the PAs-induced HSOS patients. Simultaneously, synthetic ability of liver was damaged in most of PAsinduced HSOS patients, which was revealed by the abnormality of albumin and PT. While, biomarkers of renal function indicated by urea and creatinine showed that most of the HSOS patients had normal renal function (Table 1). In addition, blood routine examination and clinical biochemistry were analyzed in the controls (BCS patients) and the results showed most of BCS patients had normal blood routine examination and normal liver function, which was demonstrated by liver function test (Table 1).

Recently, new EBMT criteria for severity grading of HSOS in adults have been established. In clinical practice, the severity of HSOS is classified by five factors containing bilirubin, liver function enzymes, weight gain, renal function and rate of change $[4,28]$. Thus, we evaluated the severity in the PAs-induced HSOS patients using new EBMT criteria. ALT exceeding 8 times the ULN was observed in $13.86 \%$ of the cases (Table 2). Besides this, similar pattern was observed in the level of AST, total bilirubin and creatinine (Table 2). Further study showed that $22(21.15 \%)$ patients with mild HSOS, $31(29.81 \%)$ patients with moderate HSOS, 21 (20.89\%) patients with severe HSOS; 30 (28.85\%) patients with very severe HSOS.

\section{Ascites analysis}

Since ascites is the most common clinical presentation of PAs-induced HSOS, some of the patients received abdominal paracentesis and the results of ascitic fluid analysis were collected. Firstly, we analyzed ascitic fluid total protein (AFTP) and serum-ascites albumin gradient (SAAG). Ascitic fluid total protein (AFTP) belonged to transudate $(<25 \mathrm{~g} / \mathrm{L})$ in $74.64 \%$ of the patients; $100 \%$ of the patients had a high SAAG $(\geq 11.0 \mathrm{~g} / \mathrm{L})$ (Table 3). Secondly, an ascitic fluid cell count and differential were analyzed. An ascitic fluid WBC counts was $134.11 \pm 135.62$; $100 \%$ of the PAs-induced HSOS patients had an ascitic polymorphonuclear (PMN) less than $0.25 \times 10^{9} / \mathrm{L}$, which indicated that no ascitic fluid infection occurred in the patients (Table 3). Finally, 41 cases received cytological examination of ascites. The results revealed that lymphocyte and mesothelial cells were main findings in the patients (Table 3). However, only 8 patient with BCS received ascitic fluid analysis in our study and the results of
Table 2 Laboratory tests including blood routine examination and clinical biochemistry at the time of baseline evaluation

\begin{tabular}{|c|c|c|}
\hline Variables & PAs-HSOS & $\mathrm{BCS}$ \\
\hline ALT <3x ULN, n/N (\%) & $64.36 \%(65 / 101)$ & $94.74 \%(54 / 57)$ \\
\hline ALT 3-8×ULN, n/N (\%) & $21.78 \%(22 / 101)$ & $3.51 \%(2 / 57)$ \\
\hline $\mathrm{ALT}>8 \times \mathrm{ULN}, \mathrm{n} / \mathrm{N}(\%)$ & $13.86 \%(14 / 101)$ & $1.75 \%(1 / 57)$ \\
\hline AST $<3 \times$ ULN, n/N (\%) & $55.88 \%(57 / 102)$ & $91.23 \%(52 / 57)$ \\
\hline AST 3-8× ULN, n/N (\%) & $35.29 \%(36 / 102)$ & $8.77 \%(5 / 57)$ \\
\hline AST > $8 \times$ ULN, n/N (\%) & $8.82 \%(9 / 102)$ & 0 \\
\hline T-BIL $<34.2 \mu \mathrm{mol} / \mathrm{L}$ & $52.94 \%(54 / 102)$ & $61.40 \%(35 / 57)$ \\
\hline T-BIL 34.2-85.5 $\mu \mathrm{mol} / \mathrm{L}, \mathrm{n} / \mathrm{N}(\%)$ & $81.37 \%(83 / 102)$ & $33.33 \%(19 / 57)$ \\
\hline T-BIL 85.5-136.8 $\mu \mathrm{mol} / \mathrm{L}, \mathrm{n} / \mathrm{N}(\%)$ & $7.84 \%(8 / 102)$ & $1.75 \%(1 / 57)$ \\
\hline T-BIL > $136.8 \mu \mathrm{mol} / \mathrm{L}, \mathrm{n} / \mathrm{N}(\%)$ & $10.78 \%(11 / 102)$ & $3.52 \%(2 / 57)$ \\
\hline $\mathrm{Cr}<1 \times \cup L N, \mathrm{n} / \mathrm{N}(\%)$ & $79.52 \%(66 / 83)$ & $96.55 \%(56 / 58)$ \\
\hline Cr 1-2×ULN, n/N(\%) & $18.07 \%(15 / 83)$ & $3.45 \%(2 / 58)$ \\
\hline $\mathrm{Cr}>2 \times \mathrm{ULN}, \mathrm{n} / \mathrm{N}(\%)$ & $2.41 \%(2 / 83)$ & 0 \\
\hline
\end{tabular}

PAs-HSOS pyrrolizidine alkaloids-induced hepatic sinusoidal obstruction syndrome, BCS Budd-Chiari syndrome, ALT alanine aminotransferase, AST aspartate aminotransferase, $T-B I L$ total bilirubin, $\mathrm{Cr}$ creatinine, ULN upper limit of normal, LLN lower limit of normal

three samples were available, thus the results of ascitic fluid analysis in BCS patients was not shown in our study.

\section{Imaging findings of contrast CT in the PAs-induced HSOS patients}

Table 4 illustrated radiographic signs seen on contrastenhanced CT in HSOS patients. Firstly, we determined the findings of pre-contrast CT in the PAs-induced HSOS patients. Ascites, global enlargement of the liver, gallbladder wall thickening, pleural effusion were common signs of PAsinduced HSOS (Table 4); while, a few of the patients (25.32\%) had splenomegaly. Secondly, we further analyzed the imaging features of contrast-enhanced CT. On contrastenhanced CT, patchy liver enhancement (93.67\%) and heterogeneous hypoattenuation (100\%) in portal-venous phase

Table $\mathbf{3}$ The results of ascitic fluid analysis

\begin{tabular}{lll}
\hline Variable & $\begin{array}{l}\text { Number patients } \\
\text { with available data }\end{array}$ \\
\hline Total protein & 71 & $21.33 \pm 7.07$ \\
Exudate $(\geq 25 \mathrm{~g} / \mathrm{L})$ & 71 & $25.35 \%(18 / 71)$ \\
SAAG $(\mathrm{g} / \mathrm{L})$ & 23 & $18.75 \pm 4.79$ \\
SAAG $(>11.0 \mathrm{~g} / \mathrm{L})$ & 23 & $100 \%(23 / 23)$ \\
Leukocyte: counts $\left(10^{6} / \mathrm{L}\right)$ & 63 & $134.11 \pm 135.62$ \\
Polymorphonuclear $\left(10^{6} / \mathrm{L}\right)$ & 57 & $28.50 \pm 36.34$ \\
Polymorphonuclear $\geq 250\left(10^{6} / \mathrm{L}\right)$ & 57 & 0 \\
Cytology & 41 & $17.07 \%(7 / 41)$ \\
Lymphocyte & 41 & $17.07 \%(7 / 41)$ \\
Mesothelial cells & 41 & $63.41 \%(26 / 41)$ \\
Lymphocyte plus mesothelial cells & 41 &
\end{tabular}


Table 4 Summary of radiological features of contrast CT in the patients with PAs-induced HSOS

\begin{tabular}{lll}
\hline Variable & $\begin{array}{l}\text { Number patients with } \\
\text { available data }\end{array}$ & Value \\
\hline Hepatomegaly & 79 & $75.95 \%$ \\
Gallbladder wall thickening & 77 & $87.01 \%$ \\
Splenomegaly & 79 & $25.32 \%$ \\
Ascites & 79 & $100 \%$ \\
Pleural effusion & 79 & $68.35 \%$ \\
Regenerative nodules & 79 & $6.33 \%$ \\
Patchy liver enhancement & 79 & $93.67 \%$ \\
Heterogeneous hypoattenuation & 79 & $100 \%$ \\
Hepatic vein narrowing (right branch) & 79 & $94.94 \%$ \\
Narrowing of inferior vena cava & 79 & $87.34 \%$ \\
\hline
\end{tabular}

were two most common radiologic findings (Table 4). Heterogeneous hypoattenuation represented heterogeneous hypoattenuated, or low-density areas; patchy liver enhancement was liver parenchyma adjacent to heterogeneous hypoattenuation appeared inhomogeneous enhancement (Fig. 2). In equilibrium phase of contrast CT, narrowing of right hepatic vein and narrowing of inferior vena cava were common signs (Table 4).

\section{Histology}

Most of PAs-induced HSOS patients did not receive percutaneous liver biopsy due to ascites, thrombocytopenia, and coagulation disorders. In addition, the expense and the unavailability of facility confined the application of transjugular biopsy in our hospitals. Thus, only 12 patients received biopsy procedure in our study, and PAsinduced SOS exhibited different pathological features. The patients received liver biopsy within 1-3 months after the initial presentation. The results of liver functional tests at the time of the liver biopsy were shown in Additional file 1: Table S1. Pathological features of the PAs-induced HSOS patients varied from early changes to subacute changes. In acute phase of PAs-induced HSOS (6 cases), liver biopsy showed massive sinusoidal dilatation and sinusoidal congestion predominantly in zone 3 accompanied by the extravasation of erythrocytes into space of Disse. In addition, we found hepatocellular necrosis, the accumulation of macrophages (Fig. 3a). In subacute stage (6 cases), important features of histopathology were the complete loss of pericentral hepatocytes, sinusoidal dilatation, the deposition of hemosiderin derived from destructive erythrocytes (Fig. 3b). The extravasation of erythrocytes and excessive deposition of collagen were not observed (Fig. 3b). The infiltration of
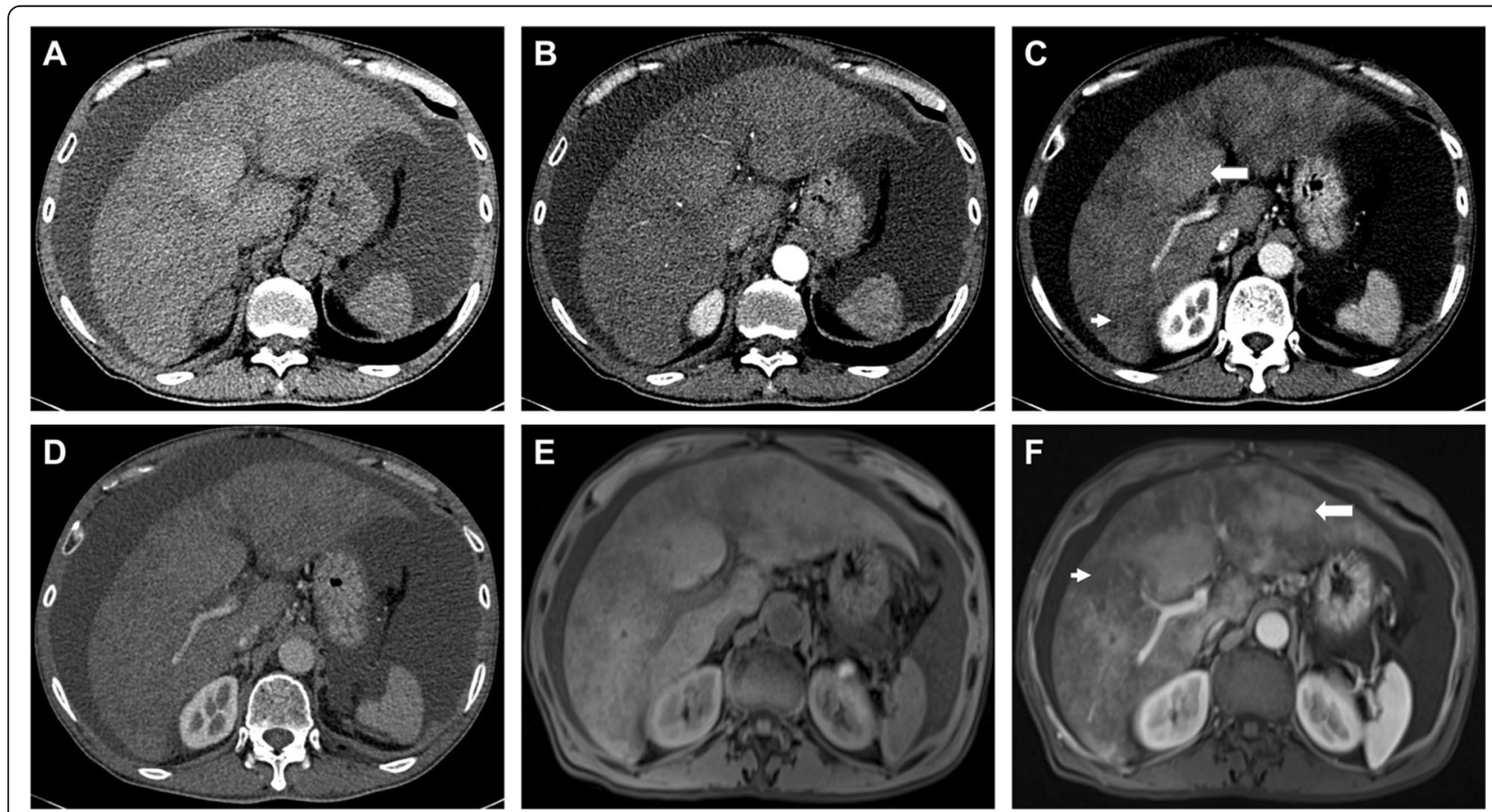

Fig. 2 Elderly male patients diagnosed with gynura segetum-induced HSOS received contrast-enhanced CT and MRI scan. a-d images of plain and contrast-enhanced CT scan; a plain CT scan; b arterial phase; c porta-venous phase; patchy liver enhancement (arrow) and heterogeneous hypoattenuation (arrowhead) were shown; $\mathbf{d}$ equilibrium phases. e-f: images of pre-contrast and portal-venous phase on dynamic contrastenhanced MRI scan;e pre-contrast MRI scan; $\mathbf{f}$ portal-venous phase of MRI scan. Heterogeneous hypointensity (arrowhead) and patchy enhancement (arrow) were shown 

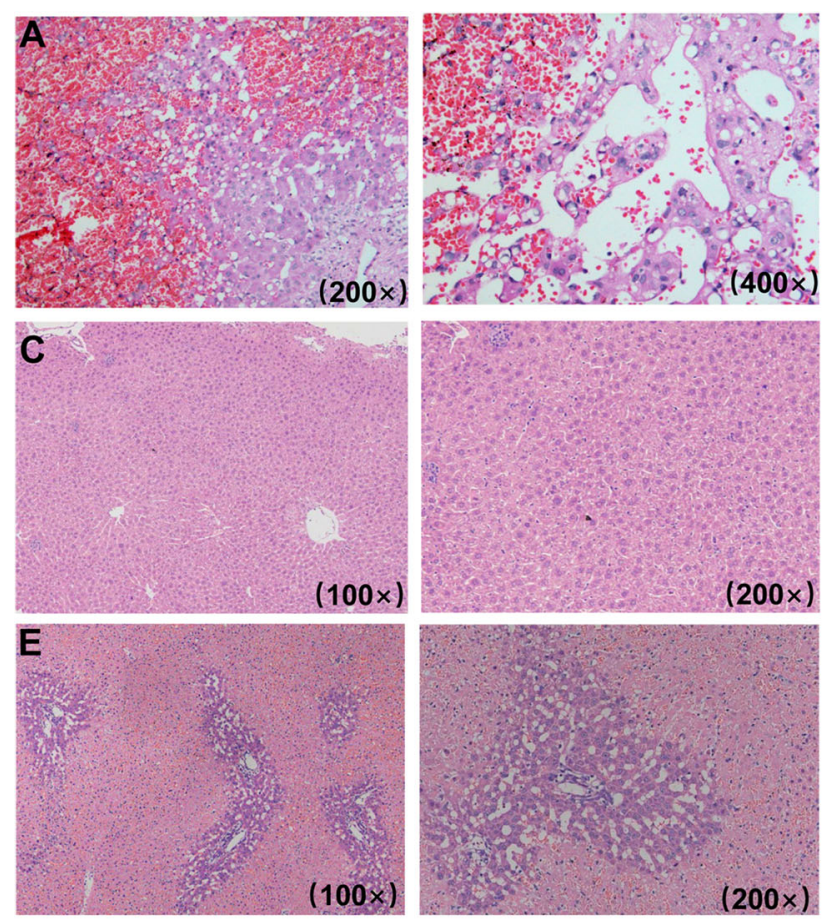
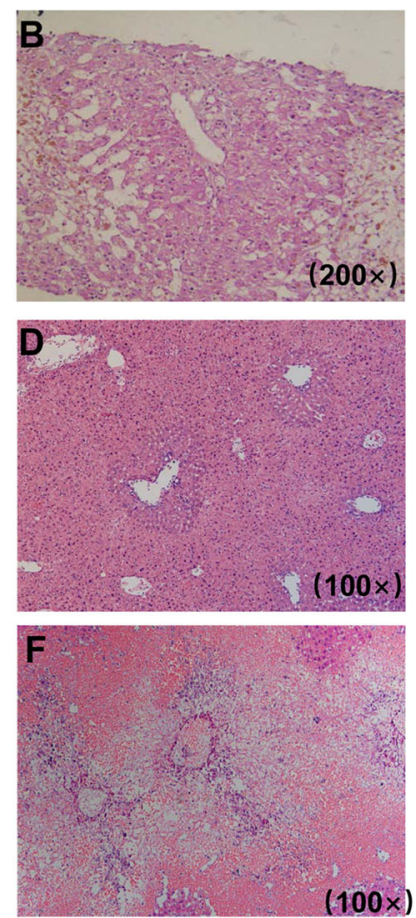
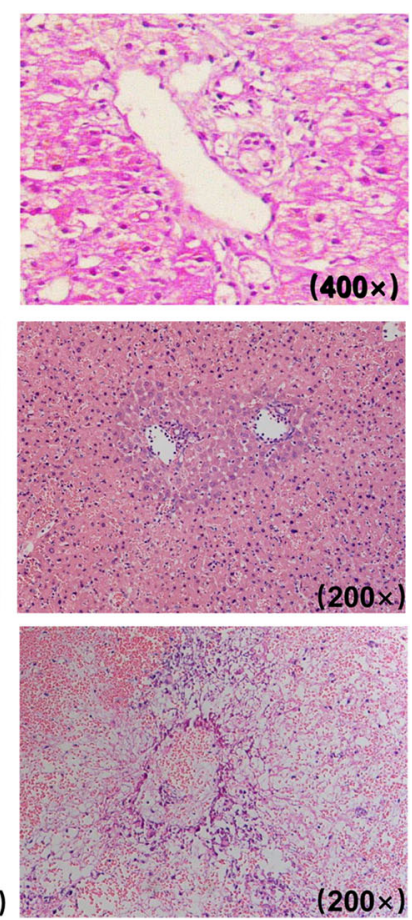

Fig. 3 Histology of the patients and rat model of PAs-induced HSOS. a: early pathological changes of the PAs-induced HSOS patients; $\mathbf{b}$ : sub-acute pathological changes of the PAs-induced HSOS patients; $\mathbf{c}$ : the livers from normal mice were stained by H\&E; $\mathbf{d}$ : $24 \mathrm{~h}$ after senecionine administration, the livers from the senecionine-treated mice were stained by H\&E; $\mathbf{e}: 48 \mathrm{~h}$ after monocrotaline administration, the livers from the rats with PAs-induced HSOS were stained by H\&E; F: 2 weeks after monocrotaline administration, pathological changes of the rats with PAs-induced HSOS

Table 5 Baseline characteristics of 117 patients with PAs-induced HSOS at the time of diagnosis

\begin{tabular}{llll}
\hline Variables & Survival Group & Death Group & $P$ Value \\
\hline Number of patients & 44 & 28 & 0.60 \\
Age, years & $59.00(47.25-63.00)$ & $61.00(51.25-65.50)$ & 0.42 \\
Male Sex; $n$ (\%) & $31.00(70.50 \%)$ & $20.00(71.40 \%)$ & 0.88 \\
Erythrocytes, $10^{12} / \mathrm{L}$ & $4.58(4.11-4.86)$ & $4.44(4.07-4.99)$ & 0.84 \\
Hemoglobin, g/L & $140.00(128.25-149.75)$ & $139.00(120.00-154.00)$ & 0.44 \\
Leukocyte, $10^{9} / \mathrm{L}$ & $5.98(4.59-7.43)$ & $7.51(5.30-8.73)$ & 0.30 \\
Platelet, $10^{9} / \mathrm{L}$ & $110.00(79.00-168.50)$ & $101.00(73.00-137.00)$ & 0.53 \\
PT, S & $16.40(15.20-18.70)$ & $17.00(16.20-18.70)$ & 0.48 \\
INR & $1.35(1.23-1.58)$ & $1.41(1.29-1.60)$ & 0.35 \\
ALT, U/L & $68.00(41.00-147.00)$ & $82.00(39.00-203.00)$ & 0.40 \\
AST, U/L & $88.00(59.00-166.00)$ & $108.00(63.00-197.00)$ & 0.07 \\
Total bilirubin, $\mu \mathrm{mol} / \mathrm{L}$ & $37.25(21.80-69.98)$ & $39.15(26.03-122.75)$ & 0.44 \\
ALB, g/l & $32.60(28.20-34.95)$ & $29.85(27.75-32.18)$ & 0.34 \\
Urea, mmol/L & $6.10(4.12-7.70)$ & $5.83(4.26-8.77)$ & 0.30 \\
Creatinine, $\mu \mathrm{mol} / \mathrm{L}$ & $77.50(64.35-87.32)$ & $81.95(66.42-97.40)$ & \\
\hline
\end{tabular}

Note: Continuous variables are presented as median (25th-75th percentiles), and categorical variables are presented as count (percentage); ${ }^{*}$ The $p$-values refer to T-test or chi-square test between patients with survival group and death group 
inflammatory cells was not obvious in acute and subacute stage.

To confirm the findings, animal models of PAsinduced HSOS were created through the administration of gynura segetum. Seneciphylline, senecionine are major compounds in Gynura segetum [24, 29]; animal models of gynura segetum-induced HSOS should be created through the administration of seneciphylline and/or senecionine. Unfortunately, seneciphylline is not available commercially. Thus, mouse model of gynura segetum-induced HSOS were established through administration of senecionine. $24 \mathrm{~h}$ after the administration, pathological features of mouse liver were similar to those of acute HSOS patients (Fig. 3d). Ten days later, senecionine-treated mice recovered by themselves. To mimics the human injury, low-dose senecionine was repeatedly injected into mice in our study. However, most of treated animals died or animal models of PAsinduced HSOS were not established unsuccessfully. Then, PAs-induced HSOS were created through the administration of monocrotaline. $48 \mathrm{~h}$ after the treatment of monocrotaline, pathological features of rat liver resembled those of acute patients with PAs-induced HSOS (Fig. 3e). At 2 weeks after the administration of monocrotaline, we found the complete loss of pericentral hepatocytes, sinusoidal dilatation and the extravasation of erythrocytes in rat liver, which indicated some of pathological changes in rat model resembled pathological features of subacute PAs-HSOS patients (Fig. 3f). In addition, chronic model of monocrotaline-induced HSOS was not created successfully through repeated administration of low-dose monocrotaline.

\section{Follow-up}

In this study, follow-up data of the patients were collected. At the end of 2-year follow-up, 38\% (44/116) of the patients (34 patients lost to follow-up, 10 patients with $<2$ years of follow-up) should be excluded; 44 patients survived, 28 patients died. Then, baseline clinical characteristics of the two groups (survival group and death group) were determined and summarized (Table 5). Unfortunately, significant differences were not observed in liver functional tests and renal function between the survival group and the death group. It probably resulted from small sample size. Thus, a prospective multi-center study should be performed to determine the prognosis of the PAs-induced HSOS patients.

\section{Discussion}

Hepatic sinusoidal obstruction syndrome (HSOS) is a rare vascular disease of the liver. Sometimes, HSOS is caused by the intake of PA-containing herbs. To get a better understanding of PAs-induced HSOS, a large sample of the patients (116 cases) with PAs-induced HSOS were enrolled and we analyzed clinical profiles of PAs-related SOS in China. In China, PAs-containing plants were soaked in liquid, such as water, tea, liquor and etc., and the patients took the liquid containing PAs; thus, we did not calculate the dosage of PAs precisely. Give this, we did not determine the correlation between total dosage of PAs and the severity of the diseases. In addition, the duration between the use of Tusanqi and first onset of clinical symptoms ranged from 2 days to 2 years; therefore, we did not distinguish acute stage from subacute/chronic diseases. Some of the patients with first-episode presentation received liver biopsy and the histology showed sub-acute pathological features. The patients with older age and male gender were frequently affected. In China, old people trust the effect of Chinese herbals, so the intake of PAscontaining plants occurred frequently to the patients with older age. Furthermore, metabolic activation of hepatotoxic PAs by cytochrome P450 (CYP) enzymes play a key role in PA-induced liver toxicities. Since hepatic expression of CYP3A1/2 in female rats is lower than that in males, male animals may be more susceptible to PAinduced toxicity than female animals [30-32].

In view of these, we determined clinical manifestations, CT findings, and pathological features of the whole PAs-induced HSOS patients. Common clinical manifestations of PAs-induced HSOS were: ascites (100\%), hyperbilirubinemia (52.94\%), and hepatomegaly (76\%). Meanwhile, a panel of liver function tests demonstrated that most of PAs-induced HSOS patients had abnormal liver function. Importantly, we assessed the severity of PAs-induced HSOS patients for the first time, which is useful to identify the patients requiring early therapeutic intervention. The resulted revealed that most of PAs-induced HSOS patients was in mild or moderate stage, which indicated that most of the patients who received routine treatment had good prognosis. While, clinical manifestations of PAs-induced HSOS mimic other liver disorders, and clinical manifestations did not provide definitive evidence in the diagnosis of PAsinduced HSOS.

In western countries, HSOS occurs most commonly in HSCT, or oxaliplatin -containing chemotherapy, and some studies described their clinical characteristics and pathological features. Previous studies demonstrated weight gain (90\%), upper abdominal pain (95\%), jaundice $(80 \%)$ were common clinical manifestations of HSOS following HSCT; only $35 \%$ of the patients had ascites [33].In addition, raised ALT, AST and ALP level were observed in the HSOS patients following HSCT [33]. In addition, some researchers reported that clinical manifestations of the patients appear to be mild or absent in the HSOS patients who received oxaliplatin-contained chemotherapy, and the level of ALT, AST, total bilirubin was in normal range [34-36]. Most of the patients were 
identified through imaging modality and/or histological examination. It indicated that clinical characteristics of HSOS correlated with the etiology of HSOS which was a determinant of the severity in the patients with HSOS.

One of clinical characteristic in PAs-induced HSOS is ascites, thus, we initially collected the results of ascitic fluid analysis. The result showed transudate $(<25 \mathrm{~g} / \mathrm{L})$ in $74.64 \%$ of the patients and high SAAG $(\geq 11.0 \mathrm{~g} / \mathrm{L})$ in $100 \%$ of the patients. Most of the patients $(95-100 \%)$ with cardiac ascites had high ascitic fluid total protein concentration (AFTP, $\geq 25 \mathrm{~g} / \mathrm{L}$ ) [37, 38]. It indicated that ascites in PAs-induced HSOS belonged to portal hypertensionrelated ascites; diagnostic efficacy of SAAG was superior to that of the exudate-transudate concept. No ascitic fluid infection occurred in PAs-induced HSOS patients, which revealed by ascitic fluid PMN counts.

The common features of dynamic CT in the PAsinduced HSOS patients contained: ascites, hepatomegaly, gallbladder wall thickening, pleural effusion, hepatic vein narrowing, patchy liver enhancement, and heterogeneous hypoattenuation. In HSOS patients after HSCT, hematomegaly, periportal edema, ascites, and narrowing of right hepatic vein were common findings of CT [39, 40]. Heterogeneity of liver parenchyma was observed in the patients of colorectal cancer who had recieved oxaliplatin-based chemotherapy [34, 41]. All these indicated that the disparity in imaging findings of contrastenhanced CT attributed to the etiology in the patients with HSOS. The etiology of HSOS is an important determinant of the severity, which may result in the difference in radiologic findings of contrast-enhanced CT. More importantly, CT sign of Budd-Chiari syndrome was analyzed, imaging features of BCS patients were described in our previous study [14]. To avoid self plagiarism, imaging signs were not provided in our manuscript.

Histological examination provided a definitive evidence of PAs-induced HSOS. However, liver biopsy was invasive and difficult to perform in routine practice due to thrombocytopenia, clotting abnormalities and extensive ascites. We firstly demonstrated PAsinduced HSOS exhibited different pathological features upon different phases. In acute stage, sinusoidal congestion, sinusoidal dilation, the necrosis of hepatocytes and the extravasation of erythrocytes in zone 3 were the characteristics of PAs-induced HSOS. In addition, macrophages infiltrated into the space of Disse, and engulfed erythrocytes. Sequentially, macrophages degraded the hemoglobin in erythrocytes, producing hemosiderin. Thus, important pathological changes in PAs-induced HSOS was the infiltration of macrophages and the deposition of hemosiderin. In sub-acute stage, pathologic examination showed complete loss of pericentral hepatocytes, sinusoidal dilatation, the deposition of pigment granules. Thus, it indicated that histological findings of PAs-induced HSOS were variable, depending upon the stages and the severity. In addition, different phases of pathological changes (early changes vs subacute changes) were observed when the patients shared similar periods from onset of illness. The varieties of pathological manifestations depend on age, the PAs dose, the period, and individual variation.

In clinical practice, PAs-induced HSOS should be considered when the patients presented with abdominal distension, jaundice, ascites, hepatomegaly. Firstly, a detailed drug-use history should be obtained. The detection of pyrrole protein adducts (PPAs) is essential for a diagnosis of PA-induced HSOS when PAs exposure is obscure. Then, dynamic enhancedcontrast CT scanning and/or MRI examination should be performed in all suspected patients. If typical signs of PA-induced HSOS are discovered, then the diagnosis can be confirmed [13, 14, 19, 42, 43]. Other chronic liver diseases, such as BCS, decompensated cirrhosis, cardiac insufficiency, should be excluded. In guideline of pyrrolizidine alkaloid-induced HSOS issued by Chinese Society of Gastroenterology Committee, supportive symptomatic treatment is the basic PA-HSOS treatment regimen. Anticoagulant therapy should be started as soon as possible in acute/subacute stage patients after ruling out contraindications TIPS should be considered when the patients do not respond to medical treatment [42]. Public education programs on potential harms of PAs-containing plants should be developed through five media (newspapers, $\mathrm{TV}$, the internet, radio, and magazines). Thus, the ingestion of PA-containing plants should be prohibited due to the toxicity of PAs.

Obviously, our study had several limitations. Firstly, it was retrospective study, not prospective study. Selection bias occurred in retrospective cohort studies. Secondly, liver biopsy was not performed in most of the patients; therefore, histological evidences were not available in most of the patients. A small sample of liver biopsy, sampling variability and patchy distribution might result in the bias. Thirdly, some of the relevant data of the patients were not available. Finally, follow-up data could not be provided since retrospective study involved 11-year span.

\section{Conclusions}

In conclusion: the PAs-induced HSOS patients displayed distinct clinical characteristics, imaging signs, and pathological features compared with HSOS associated with HSCT and oxaliplatin-containing chemotherapy. Further studies should be performed to explore histological changes based on large samples, develop suitable therapeutic strategies, and investigate the prognosis in future. 


\section{Supplementary information}

Supplementary information accompanies this paper at https://doi.org/10. 1186/s12876-020-1180-0.

Additional file 1: Table S1. The results of liver functional test at the time of the liver biopsy

\section{Abbreviations}

AFTP: Ascitic fluid total protein; ALP: Alkaline phosphatase; ALT: Alanine aminotransferase; AST: Aspartate aminotransferase; BCS: Budd-Chiari syndrome; CT: Computed tomography; DCE: Dynamic contrast-enhanced; HSCT: Hematopoietic stem cell transplantation; HSOS: Hepatic sinusoidal obstruction syndrome; LLN: Lower limit of normal; MRI: Magnetic resonance imaging; PA: Pyrrolizidine alkaloid; PMN: Polymorphonuclear; PT: Prothrombin time; SAAG: Serum ascites albumin gradient; ULN: Upper limit of normal; VOD: Veno-occlusive disease; WBC: White blood cell; $\gamma$-GT: $\gamma$-glutamyl transpeptidase

\section{Acknowledgements}

We thank Dr. Keshu Xu, Mrs. Jin Ye, Mrs. Ling Yang, Mrs. Xiaoli Pan (Division of Gastroenterology, Union Hospital, Tongji Medical College, HUST) for helpful suggestions in data collection, and Dr. Xin Li (Department of Radiology, Union Hospital, Tongji Medical College, HUST) for kind assistance in the collection of CT images.

\section{Authors' contributions}

YS designed the study; $X R, C L, L M, X Y$, and DX collected data; $X R, X K$, and TG reviewed $C T$ imaging; $H G$ reviewed patient's histology; $F L, X R$, YS analyzed the data, reviewed the chart, and interpreted the data; FL, XR, YS wrote the paper. All authors have read and approved the manuscript.

\section{Funding}

This work was partially supported by National Natural Science Foundation of China $(81570555,81270506)$ and Clinical Research Physician Program of Tongji Medical College, HUST (2017). The funding bodies played no role in the design of the study, the collection, analysis and interpretation of data or in the writing of the manuscript.

\section{Availability of data and materials}

The raw data generated and analyzed in the current study are not publicly available due to appropriate protection of patient personal information but are available from the corresponding author on reasonable request.

\section{Ethics approval and consent to participate}

The study was approved by the ethics committee of Tongii Medical College, Huazhong University of Science and Technology (IORG0003571) prior to data collection. All procedures followed were in accordance with the ethical standards of the responsible committee on human experimentation (institutional and national) and with the Helsinki Declaration of 1975, as revised in 2008. This study is a retrospective analysis, it did not include any human trial, and as such no informed consent from patients was needed.

\section{Consent for publication}

Not applicable.

\section{Competing interests}

The authors declare that they have no competing interests.

\section{Author details}

${ }^{1}$ Institute of Hematology, Union Hospital, Tongji Medical College, Huazhong University of Science and Technology, Wuhan 430022, China. ${ }^{2}$ Division of Gastroenterology, Union Hospital, Tongji Medical College, Huazhong University of Science and Technology, Wuhan 430022, China. ${ }^{3}$ Institute of Organ Transplantation, Tongji Hospital, Tongji Medical College, Huazhong University of Science and Technology, Wuhan 430030, China. ${ }^{4}$ Department of Infectious Diseases, Tongji Hospital, Tongji Medical College, Huazhong University of Science and Technology, Wuhan 430030, China. ${ }^{5}$ Department of Radiology, Union Hospital, Tongji Medical College, Huazhong University of Science and Technology, Wuhan 430022, China.
Received: 17 June 2019 Accepted: 28 January 2020

Published online: 04 February 2020

\section{References}

1. DeLeve LD, Valla DC, Garcia-Tsao G. Vascular disorders of the liver. Hepatology. 2009;49(5):1729-64.

2. Plessier A, Rautou PE, Valla DC. Management of hepatic vascular diseases. J Hepatol. 2012;56(Suppl 1):S25-38

3. Valla DC, Cazals-Hatem D. Sinusoidal obstruction syndrome. Clin Res Hepatol Gastroenterol. 2016;40(4):378-85.

4. Fan CQ, Crawford JM. Sinusoidal obstruction syndrome (hepatic venoocclusive disease). J Clin Exp Hepatol. 2014;4(4):332-46.

5. Blostein MD, Paltiel OB, Thibault A, Rybka WB. A comparison of clinical criteria for the diagnosis of veno-occlusive disease of the liver after bone marrow transplantation. Bone Marrow Transplant. 1992;10(5):439-43.

6. Mohty M, Malard F, Abecassis M, Aerts E, Alaskar AS, Aljurf M, Arat M, Bader $P$, Baron F, Bazarbachi A, et al. Revised diagnosis and severity criteria for sinusoidal obstruction syndrome/veno-occlusive disease in adult patients: a new classification from the European Society for Blood and Marrow Transplantation. Bone Marrow Transplant. 2016:51(7):906-12.

7. Gao H, Ruan JQ, Chen J, Li N, Ke CQ, Ye Y, Lin G, Wang JY. Blood pyrroleprotein adducts as a diagnostic and prognostic index in pyrrolizidine alkaloid-hepatic sinusoidal obstruction syndrome. Drug Design Dev Ther. 2015;9:4861-8.

8. Wang JY, Gao H. Tusanqi and hepatic sinusoidal obstruction syndrome. J Dig Dis. 2014;15(3):105-7.

9. Lin G, Wang JY, Li N, Li M, Gao H, Ji Y, Zhang F, Wang H, Zhou Y, Ye Y, et al. Hepatic sinusoidal obstruction syndrome associated with consumption of Gynura segetum. J Hepatol. 2011;54(4):666-73.

10. Yang XQ, Ye J, Li X, Li Q, Song YH. Pyrrolizidine alkaloids-induced hepatic sinusoidal obstruction syndrome: pathogenesis, clinical manifestations, diagnosis, treatment, and outcomes. World J Gastroenterol. 2019;25(28): 3753-63.

11. Chojkier M. Hepatic sinusoidal-obstruction syndrome: toxicity of pyrrolizidine alkaloids. J Hepatol. 2003;39(3):437-46.

12. Wang X, Qi X, Guo X. Tusanqi-related sinusoidal obstruction syndrome in China: a systematic review of the literatures. Medicine. 2015;94(23):e942.

13. Li X, Yang X, Xu D, Li Q, Kong X, Lu Z, Bai T, Xu K, Ye J, Song Y. Magnetic resonance imaging findings in patients with Pyrrolizidine alkaloid-induced hepatic sinusoidal obstruction syndrome. Clin Gastroenterol Hepatol. 2017; 15(6):955-7.

14. Kan X, Ye J, Rong X, Lu Z, Li X, Wang Y, Yang L, Xu K, Song Y, Hou X. Diagnostic performance of contrast-enhanced $C T$ in Pyrrolizidine alkaloids-induced hepatic sinusoidal obstructive syndrome. Sci Rep. 2016:6:37998

15. Chalasani NP, Hayashi PH, Bonkovsky HL, Navarro VJ, Lee WM, Fontana RJ. Practice parameters Committee of the American College of G: ACG clinical guideline: the diagnosis and management of idiosyncratic drug-induced liver injury. Am J Gastroenterol. 2014;109(7):950-66 quiz 967.

16. Danan G, Benichou C. Causality assessment of adverse reactions to drugs--I. a novel method based on the conclusions of international consensus meetings: application to drug-induced liver injuries. J Clin Epidemiol. 1993; 46(11):1323-30.

17. Gao H, Li N, Wang JY, Zhang SC, Lin G. Definitive diagnosis of hepatic sinusoidal obstruction syndrome induced by pyrrolizidine alkaloids. J Dig Dis. 2012:13(1):33-9.

18. Yang M, Ruan J, Fu PP, Lin G. Cytotoxicity of pyrrolizidine alkaloid in human hepatic parenchymal and sinusoidal endothelial cells: firm evidence for the reactive metabolites mediated pyrrolizidine alkaloid-induced hepatotoxicity. Chem Biol Interact. 2016:243:119-26.

19. Guo T, Li X, Yang X, Kong X, Liu H, Bai T, Xu K, Ye J, Song Y. Gadoxetic acidenhanced Hepatobiliary-phase magnetic resonance imaging for Pyrrolizidine alkaloid-induced hepatic sinusoidal obstruction syndrome and association with liver function. Sci Rep. 2019;9(1):1231.

20. Du L, Zhu S, Lu Z, Xu T, Bai T, Xu D, Wei X, Li J, Xu K, Ye J, et al. Ascitic cholesterol is superior to serum-ascites albumin gradient in the detection of non-portal hypertensive ascites and the diagnosis of mixed ascites. Aliment Pharmacol Ther. 2019;49(1):91-8.

21. Aydinli M, Bayraktar Y. Budd-Chiari syndrome: etiology, pathogenesis and diagnosis. World J Gastroenterol. 2007;13(19):2693-6. 
22. DeLeve LD, McCuskey RS, Wang X, Hu L, McCuskey MK, Epstein RB, Kanel GC. Characterization of a reproducible rat model of hepatic veno-occlusive disease. Hepatology. 1999;29(6):1779-91.

23. Yang X, Wang H, Ni HM, Xiong A, Wang Z, Sesaki H, Ding WX, Yang L. Inhibition of Drp1 protects against senecionine-induced mitochondriamediated apoptosis in primary hepatocytes and in mice. Redox Biol. 2017; 12:264-73.

24. Yang M, Ruan J, Gao H, Li N, Ma J, Xue J, Ye Y, Fu PP, Wang J, Lin G. First evidence of pyrrolizidine alkaloid N-oxide-induced hepatic sinusoidal obstruction syndrome in humans. Arch Toxicol. 2017:91(12):3913-25.

25. Nakamura K, Hatano E, Miyagawa-Hayashino A, Okuno M, Koyama Y, Narita M, Seo S, Taura K, Uemoto S. Soluble thrombomodulin attenuates sinusoidal obstruction syndrome in rat through suppression of high mobility group box 1. Liver Int. 2014;34(10):1473-87.

26. Nakamura K, Hatano E, Narita M, Miyagawa-Hayashino A, Koyama Y, Nagata H, Iwaisako K, Taura K, Uemoto S. Sorafenib attenuates monocrotalineinduced sinusoidal obstruction syndrome in rats through suppression of JNK and MMP-9. J Hepatol. 2012;57(5):1037-43.

27. Edgar JA, Molyneux RJ, Colegate SM. Pyrrolizidine alkaloids: potential role in the etiology of cancers, pulmonary hypertension, congenital anomalies, and liver disease. Chem Res Toxicol. 2015:28(1):4-20.

28. Chao N. How I treat sinusoidal obstruction syndrome. Blood. 2014;123(26): 4023-6.

29. Fang L, Xiong A, Yang X, Cheng W, Yang L, Wang Z. Mass-spectrometrydirected analysis and purification of pyrrolizidine alkaloid cis/trans isomers in Gynura japonica. J Sep Sci. 2014;37(15):2032-8.

30. Lin G, Cui YY, Liu XQ, Wang ZT. Species differences in the in vitro metabolic activation of the hepatotoxic pyrrolizidine alkaloid clivorine. Chem Res Toxicol. 2002;15(11):1421-8.

31. Lin G, Cui YY, Liu XQ. Gender differences in microsomal metabolic activation of hepatotoxic clivorine in rat. Chem Res Toxicol. 2003;16(6):768-74.

32. Lin G, Tang J, Liu XQ, Jiang Y, Zheng J. Deacetylclivorine: a gender-selective metabolite of clivorine formed in female Sprague-Dawley rat liver microsomes. Drug Metab Dispos. 2007;35(4):607-13.

33. Kami M, Mori S, Tanikawa S, Akiyama H, Onozawa Y, Tanaka T, Okamoto R, Maeda Y, Sasaki T, Kaku H, et al. Risk factors for hepatic veno-occlusive disease after bone marrow transplantation: retrospective analysis of 137 cases at a single institution. Bone Marrow Transplant. 1997;20(5):397-402.

34. Han NY, Park BJ, Kim MJ, Sung DJ, Cho SB. Hepatic parenchymal heterogeneity on contrast-enhanced $C T$ scans following Oxaliplatin-based chemotherapy: natural history and association with clinical evidence of sinusoidal obstruction syndrome. Radiology. 2015;276(3):766-74.

35. Shin NY, Kim MJ, Lim JS, Park MS, Chung YE, Choi JY, Kim KW, Park YN. Accuracy of gadoxetic acid-enhanced magnetic resonance imaging for the diagnosis of sinusoidal obstruction syndrome in patients with chemotherapytreated colorectal liver metastases. Eur Radiol. 2012;22(4):864-71.

36. Liu F, Cao X, Ye J, Pan X, Kan X, Song Y. Oxaliplatin-induced hepatic sinusoidal obstruction syndrome in a patient with gastric cancer: a case report. Mol Clin Oncol. 2018:8(3):453-6.

37. Farias $A Q$, Silvestre $O M$, Garcia-Tsao G, da Costa Seguro LF, de Campos Mazo DF, Bacal F, Andrade JL, Goncalves LL, Strunz C, Ramos DS, et al. Serum B-type natriuretic peptide in the initial workup of patients with new onset ascites: a diagnostic accuracy study. Hepatology. 2014;59(3):1043-51.

38. Runyon BA, Committee APG. Management of adult patients with ascites due to cirrhosis: an update. Hepatology. 2009;49(6):2087-107.

39. Erturk SM, Mortele KJ, Binkert CA, Glickman JN, Oliva MR, Ros PR, Silverman SG. CT features of hepatic venoocclusive disease and hepatic graft-versushost disease in patients after hematopoietic stem cell transplantation. AJR Am J Roentgenol. 2006;186(6):1497-501.

40. van den Bosch MA, van Hoe L. MR imaging findings in two patients with hepatic veno-occlusive disease following bone marrow transplantation. Eur Radiol. 2000;10(8):1290-3.

41. Han NY, Park BJ, Sung DJ, Kim MJ, Cho SB, Lee CH, Jang YJ, Kim SY, Kim DS, Um SH, et al. Chemotherapy-induced focal hepatopathy in patients with gastrointestinal malignancy: gadoxetic acid--enhanced and diffusionweighted MR imaging with clinical-pathologic correlation. Radiology. 2014; 271(2):416-25

42. Zhuge Y, Liu Y, Xie W, Zou X, Xu J, Wang J, Chinese Society of Gastroenterology Committee of Hepatobiliary D. Expert consensus on the clinical management of pyrrolizidine alkaloid-induced hepatic sinusoidal obstruction syndrome. J Gastroenterol Hepatol. 2019;34(4):634-42.
43. Zhou H, Wang YX, Lou HY, Xu XJ, Zhang MM. Hepatic sinusoidal obstruction syndrome caused by herbal medicine: $C T$ and MRI features. Kor J Radiol. 2014;15(2):218-25.

\section{Publisher's Note}

Springer Nature remains neutral with regard to jurisdictional claims in published maps and institutional affiliations.
Ready to submit your research? Choose BMC and benefit from:

- fast, convenient online submission

- thorough peer review by experienced researchers in your field

- rapid publication on acceptance

- support for research data, including large and complex data types

- gold Open Access which fosters wider collaboration and increased citations

- maximum visibility for your research: over $100 \mathrm{M}$ website views per year

At $\mathrm{BMC}$, research is always in progress.

Learn more biomedcentral.com/submissions 